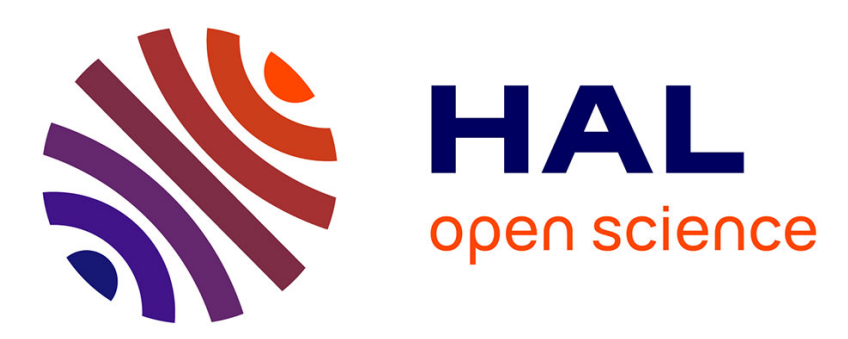

\title{
Revisiting the constitutive equations of unsaturated porous solids using a Lagrangian saturation concept \\ O Coussy
}

\section{To cite this version:}

O Coussy. Revisiting the constitutive equations of unsaturated porous solids using a Lagrangian saturation concept. International Journal for Numerical and Analytical Methods in Geomechanics, 2007, 31 (15), pp.1675 - 1694. 10.1002/nag.613 . hal-00359952

\section{HAL Id: hal-00359952 https://hal.science/hal-00359952}

Submitted on 11 Jul 2017

HAL is a multi-disciplinary open access archive for the deposit and dissemination of scientific research documents, whether they are published or not. The documents may come from teaching and research institutions in France or abroad, or from public or private research centers.
L'archive ouverte pluridisciplinaire HAL, est destinée au dépôt et à la diffusion de documents scientifiques de niveau recherche, publiés ou non, émanant des établissements d'enseignement et de recherche français ou étrangers, des laboratoires publics ou privés. 


\title{
Revisiting the constitutive equations of unsaturated porous solids using a Lagrangian saturation concept
}

\author{
O. Coussy*, $\dagger$ \\ UMR Navier, ENPC, 6-8 Av. Blaise Pascal, Cité Descartes, Champs-sur-Marne, 77455 Marne-la-Valle \\ Cedex 277420, Champs-sur-Marne, France
}

\begin{abstract}
SUMMARY
This paper aims at revisiting the constitutive equations of unsaturated porous solids at the light of a Lagrangian saturation concept. By referring the currently wetted porous volume to the reference configuration, the Lagrangian saturation is the state variable associated with the interfacial energy changes only, irrespective of the elastic energy required for deforming the solid matrix. The Lagrangian saturation concept provides the basis of a generic approach to the theory of poroelastoplasticity in unsaturated conditions. We successively examine the case where the saturating fluids occupy disconnected networks and the case where the networks are connected so that the saturating fluids can invade the porous solid or recede from it. The analysis provides the restricted situations where the averaged pore pressure may play the role of an effective pore pressure. Copyright (C) 2007 John Wiley \& Sons, Ltd.
\end{abstract}

Received 5 September 2006; Revised 8 February 2007; Accepted 17 February 2007

KEY WORDS: unsaturated; thermodynamics; poroelasticity; plasticity; interfacial energy; effective stress

\section{INTRODUCTION}

The understanding of the mechanical behaviour of unsaturated porous solids is relevant to various materials and applications. Examples are many: cement-based materials in civil engineering, woods in building industry, rocks and soils in geosciences, gels in physical chemistry, living tissues and bones in biomechanics, etc. Although most of the physical phenomena to which these materials are subjected are the same, namely consolidation, temperature changes, drying shrinkage, cryosuction, osmotic phenomena, etc., similar early approaches have been often separately elaborated, depending on the context of applications. We may, for instance, refer to the celebrated pioneering

\footnotetext{
*Correspondence to: O. Coussy, UMR Navier, ENPC, 6-8 Av. Blaise Pascal, Cité Descartes, Champs-sur-Marne, 77455 Marne-la-Valle Cedex 277420, Champs-sur-Marne, France.

†E-mail: olivier.coussy@mail.enpc.fr
} 
works of Terzaghi in soil mechanics [1], of Biot in rocks mechanics [2], and of Powers in cementbased materials science [3].

Through the years a global consensus has emerged that, whatever the targeted applications, the mechanics of fully or partially saturated porous media may be successfully addressed with the general tools of continuum mechanics. The difficulty of extending continuum mechanics to fluid-infiltrated porous media resides in the two-phase character of the medium. Two steps have to be distinguished. The first step is to extend the macroscopic balance laws of continuum mechanics, relative to the mass, the momentum, the energy, and the entropy, to a multiphase porous medium. Nowadays, based on extensive works this goal may be considered as satisfactorily achieved. The most convincing approach is to work out averaging methods. Starting from the microscopic scale, that is, from the scale of the material points within the constitutive phases themselves, they provide the field equations which can be ultimately associated with a multiphase continuum. Without pretending to the exhaustiveness, we refer the reader to [4-11]. Another close intermediary viewpoint is to express the balance laws by starting from the mesoscopic scale, that is, from the scale of the constitutive phases already considered as material points. This viewpoint has been initiated early by Biot $[2,12]$, and extensively developed in [13-15]. It has been shown that both viewpoints are finally equivalent [16].

Once the balance laws are derived, the second step is to extend the usual constitutive equations of solids to fluid-infiltrated porous solids. The cornerstone of this extension is the multiphase Clausius-Duhem inequality resulting from the first and the second laws of thermodynamics relative to the macroscopic scale of the elementary representative volume. This inequality permits the determination of the couples of state variables which are energy conjugate. This determination is the basis of the derivation of the general form that the constitutive equations have to take for each wide class of behaviours (elastic, plastic, etc.), in order to be consistent regarding the transformations of energy whatever the scale at which these transformations actually occur. Once the constitutive equations derived from this non-questionable thermodynamic basis, micro-macro considerations [11] may then be subsequently and independently used for providing assessments of the macroscopic properties these constitutive equations involve. In saturated conditions, the theory of poroelasticity, as initiated by Biot [2] for infinitesimal transformations, and its natural extension to poroplasticity, are now widely accepted as the consistent extension to fluid-infiltrated porous solids of the usual theory of elastoplasticity of solids (see [17, 18], for an introduction to poroelasticity, and among others $[15,19]$ for irreversible behaviours). In addition to the macroscopic stress, the pore pressure plays the role of an extra loading parameter, acting on the internal solid walls of the porous solid. The pore pressure energy conjugate variable is then identified with the change in porosity.

The extension of saturated constitutive equations to unsaturated conditions is still a matter of debate. In order to recover the familiar saturated conditions, this extension is often worked out through the quest of a unique 'effective' pore pressure accounting equivalently for the effects of the pressure of all the saturating fluids acting separately on the internal solid walls. Among other works, we refer the reader to [20-24]. The natural candidate for this effective pore pressure is the space-averaged pore pressure. The averaged pressure is the sum of the fluid pressures weighted by the pore volume fraction related to each fluid. The pore volume fraction used in the summation is generally defined with regard to the current overall porous volume. Such a pore volume fraction may be coined as the Eulerian saturation since it refers to the current configuration. Since the current configuration undergoes a deformation, there is a contribution of the current pore deformation to the Eulerian saturation. As a consequence, an infinitesimal change in the Eulerian saturation does 
not only relate to the further invasion of the porous volume by the corresponding fluid, but also to the further deformation of the porous volume already invaded by the same fluid. Irrespective of the assumption of infinitesimal transformations, this precludes the use of the Eulerian saturation from providing an energy balance accounting for the energy involved in the invasion process separately from that involved in the deformation process. The purpose of this paper is to overcome this difficulty by using a Lagrangian saturation concept recently introduced in $[25,26]$ to address the mechanics of confined crystallization. In contrast to the Eulerian saturation, the Lagrangian saturation refers the current saturation process to the initial configuration and thereby is irrespective of the pore deformation. This concept will reveal as being efficient to separately capture the case of disconnected networks, where the saturating fluids remain in contact with the same internal solid walls, and the case of connected networks, where new solid-fluid interfaces are created during the drainage or the imbibition of the porous solid.

In order to introduce the general energy viewpoint and concepts extensively used later on, we first revisit the familiar case of saturated porous solids, showing that the theory holds irrespective of the ideal or non-ideal character of the saturating solution. In the second section, we extend the approach to unsaturated conditions where the saturating fluids occupy disconnected networks. We show that the validity of using the space-averaged pore pressure as an effective pore pressure is founded on the assumption of the iso-deformation of the porous networks. This assumption remains questionable for most of the natural porous materials so that the validity of the effective pore pressure concept turns out to be limited. In the last section, we show how the Lagrangian saturation concept is the key to the extension of the previous analysis to the more difficult case of connected networks, by capturing the particular role played by the change in the energy associated with the solid-fluid interface in the formulation of the constitutive equations of unsaturated porous solids. The relevance of using the net stress and the capillary pressure as effective stresses, which has been proved to be appropriate in the specific context of soil mechanics [27], can be finally explored in this more general context.

\section{REVISITING SATURATED POROELASTOPLASTICITY}

The thermodynamics of heterogeneous substances goes back to the works of Gibbs [28] and Duhem [29]. They have furnished the long-celebrated Gibbs-Duhem equation extensively used in the thermodynamics of mixtures. Affiliated with the same energy approach, the thermodynamics of non-rigid saturated porous materials have been initiated by Biot [12], allowing to extend the Clausius-Duhem inequality to porous solids $[13,15]$. The Clausius-Duhem inequality is the cornerstone of any energy approach to the constitutive equations of materials. This section intends to briefly revisit the thermodynamics of saturated porous solids in order to provide the natural framework to further explore unsaturated conditions.

\subsection{Lagrangian and Eulerian porosity: solid matrix incompressibility}

Consider an infinitesimal representative element of porous solid extracted from a porous continuum. This element of porous solid consists of both a solid matrix (the solid mineral for a rock, the solid grains for a soil), and a connected porous volume which may be filled by one (saturated conditions) or several fluids (unsaturated conditions). In the undeformed reference configuration the overall volume of the porous solid element is $\mathrm{d} \Omega_{0}$. Its porous volume is $\phi_{0} \times \mathrm{d} \Omega_{0}$, where $\phi_{0}$ stands for the 
(initial) porosity. At current time $t$ the porous volume is $\phi \times \mathrm{d} \Omega_{0}$, where $\phi$ stands for the current porosity. Since porosity $\phi$ refers the current porous volume to the reference volume $\mathrm{d} \Omega_{0}, \phi$ can be coined as the Lagrangian porosity [15]. Let $\varphi$ then be defined as the change in the Lagrangian porosity, that is

$$
\varphi=\phi-\phi_{0}
$$

so that $\varphi \times \mathrm{d} \Omega_{0}$ captures the overall change of the porous volume with regard to the reference configuration. As any strain related to a (porous) solid, $\varphi$ is also a Lagrangian variable.

The current porous volume may also be referred to the current overall volume $d \Omega$ of the porous solid element. The related porosity is the Eulerian porosity $n$ defined by

$$
n \times \mathrm{d} \Omega=\phi \times \mathrm{d} \Omega_{0}
$$

In all that follows we will restrict ourselves to infinitesimal strains, the extension to finite transformations presenting no specific difficulty with regard to standard continuum mechanics (see [15]). If $\varepsilon$ denotes the overall volumetric strain of the porous solid, the current overall volume $\mathrm{d} \Omega$ is then linked to the initial volume $\mathrm{d} \Omega_{0}$ by

$$
\mathrm{d} \Omega=(1+\varepsilon) \mathrm{d} \Omega_{0}
$$

so that

$$
\phi=(1+\varepsilon) n
$$

From (4) one might think that the Lagrangian porosity $\phi$ and the Eulerian porosity $n$ may be indifferently used under the assumption of infinitesimal strains where $\varepsilon \ll 1$. This is not correct with regard to their infinitesimal variations. In fact, since $n_{0}=\phi_{0}$ and that $\varepsilon \mathrm{d} n$ is a second-order term, from (1) and (4) we get

$$
\mathrm{d} \phi=\mathrm{d} \varphi \simeq \mathrm{d} n+\phi_{0} \mathrm{~d} \varepsilon
$$

The strain work per unit of initial volume $\mathrm{d} \Omega_{0}$ related to the porous solid associated with the pore pressure $p$ applying on the internal solid walls of the porous network is $p \mathrm{~d} \phi=p \mathrm{~d} \varphi \neq p \mathrm{~d} n$. The use of expression $p \mathrm{~d} n$ instead of $p \mathrm{~d} \phi$ would mislead to neglect the term $p \phi_{0} \mathrm{~d} \varepsilon$ which, according to (5), has the same order of magnitude as $p \mathrm{~d} n$, even under the assumption of infinitesimal strains. As a conclusion, even in the usual case of infinitesimal strains, a distinction has still to be made between the Lagrangian porosity and the Eulerian porosity, whereas the use of the former must be preferred.

The overall volumetric strain $\varepsilon$ of the porous solid results from both the volumetric strain $\varepsilon_{\mathrm{s}}$ of its solid part, i.e. the solid matrix, and from the change $\varphi$ of the Lagrangian porosity. The balance of all the volume changes [15] provides the relation

$$
\varepsilon=\left(1-\phi_{0}\right) \varepsilon_{\mathrm{s}}+\varphi
$$

For some usual porous solids, as for instance soils, the solid matrix is formed of incompressible grains so that $\varepsilon_{\mathrm{S}}=0$ and the previous relation reduces to

$$
\varepsilon=\varphi
$$




\subsection{Gibbs-Duhem equality for the saturating solution}

In this first section, the porous volume is assumed to remain fully saturated by a solution. However, the solution is assumed to be a mixture formed of several species. The species are referred to by index $\alpha=1,2 \ldots$. Let then $n_{\alpha} \times \mathrm{d} \Omega_{0}$ be the number of moles of the species $\alpha$ currently present in the porous volume occupied by the solution, so that $n_{\alpha}$ represents the (Lagrangian) macroscopic or apparent molar density of species $\alpha$. Let $p$ be the pressure of the solution and $\mu_{\alpha}$ the current molar chemical potential of species $\alpha$. The isothermal Gibbs-Duhem equality related to the solution can be written in the form

$$
\phi \mathrm{d} p-n_{\alpha} \mathrm{d} \mu_{\alpha}=0
$$

\subsection{State equations of saturated poroelasticity}

During its physical evolution the system $d \Omega_{0}$ exchanges moles of species $\alpha$ with the adjacent infinitesimal systems forming the two-phase continuum so that $\mathrm{d} \Omega_{0}$ is an open thermodynamic system. To derive the associated Clausius-Duhem equality, let then $\sigma_{i j}$ be the overall stress components to which the system $\mathrm{d} \Omega_{0}$ is subjected; let also $\varepsilon_{i j}$ be the current overall infinitesimal strain components capturing the deformation with regard to the reference configuration. Finally, let $F_{\text {op }}$ be the overall Helmholtz free energy of the open element per unit of its initial volume $\mathrm{d} \Omega_{0}$, that is, including both the free energy of the solid part of the porous solid and that of the saturating solution. The first and the second laws of thermodynamics applied to the open system $\mathrm{d} \Omega_{0}$ between times $t$ and $t+\mathrm{d} t$ combine to provide the isothermal Clausius-Duhem inequality in the form

$$
\sigma_{i j} \mathrm{~d} \varepsilon_{i j}+\sum_{\alpha} \mu_{\alpha} \mathrm{d} n_{\alpha}-\mathrm{d} F_{\text {op }} \geqslant 0
$$

In addition to the familiar strain work $\sigma_{i j} \mathrm{~d} \varepsilon_{i j}$ related to the deformation of the infinitesimal element $\mathrm{d} \Omega_{0}$, the added term $\mu_{\alpha} \mathrm{d} n_{\alpha}$ is the free energy supply associated with the change in the molar content $\mathrm{d} n_{\alpha}$ of species $\alpha$. Inequality (9) states that the difference between the overall free energy supply whatever its form, and the infinitesimal free energy $\mathrm{d} F_{\text {op }}$ eventually stored by the open system, cannot be negative since it is possibly dissipated into heat.

From the energy balance (9) related to the open system $d \Omega_{0}$, we want now to extract the energy balance related to the porous solid only, the porous solid being the material obtained by removing the bulk solution and whose internal solid walls are subjected to pressure $p$ of the solution. Let then $F$ be the Helmholtz free energy of the porous solid per unit of its initial volume $\mathrm{d} \Omega_{0}$. As a direct consequence of the additive character of the free energy, the Helmholtz free energy of the porous solid can be written in the form

$$
F=F_{\text {op }}-\left(\sum_{\alpha} n_{\alpha} \mu_{\alpha}-\phi p\right)
$$

Using (8) and (10) into (9), we get

$$
\sigma_{i j} \mathrm{~d} \varepsilon_{i j}+p \mathrm{~d} \phi-\mathrm{d} F \geqslant 0
$$

Energy balance (11) holds irrespective of the solution composition and, thereby, of its ideal or non-ideal character. Actually, it relates to the porous solid only, the extra term $p \mathrm{~d} \phi$ accounting for the work supplied to the porous solid through its internal solid walls whatever the origin of 
the pore pressure $p$. Owing to the additive character of energy, $F$ is also the free energy of the solid matrix forming the solid part of the porous solid. In order to emphasize this aspect, referring to the solid matrix by index $s$, we write

$$
F=F_{\mathrm{s}}
$$

Use of (1) and (12) allows us to rewrite (11) in the equivalent form

$$
\sigma_{i j} \mathrm{~d} \varepsilon_{i j}+p \mathrm{~d} \varphi-\mathrm{d} F_{\mathrm{s}} \geqslant 0
$$

For a porous elastic solid (poroelastic solid) there is no dissipation and (13) reduces to an equality

$$
\sigma_{i j} \mathrm{~d} \varepsilon_{i j}+p \mathrm{~d} \varphi-\mathrm{d} F_{\mathrm{s}}=0
$$

From (14) we finally obtain the state equations of saturated poroelasticity in the form

$$
\sigma_{i j}=\frac{\partial F_{\mathrm{s}}}{\partial \varepsilon_{i j}}, \quad p=\frac{\partial F_{\mathrm{s}}}{\partial \varphi}
$$

\subsection{Linear poroelasticity and poroelastic properties}

Alternatively, we can introduce the Legendre transform $W_{\mathrm{s}}$ of $F_{\mathrm{s}}$ with regard to $\varphi$

$$
W_{\mathrm{s}}=F_{\mathrm{s}}-\varphi p
$$

and rewrite (13) in the form

$$
\sigma_{i j} \mathrm{~d} \varepsilon_{i j}-\varphi \mathrm{d} p-\mathrm{d} W_{\mathrm{s}}=0
$$

From (17) we now derive the state equations of saturated poroelasticity in the form

$$
\sigma_{i j}=\frac{\partial W_{\mathrm{s}}}{\partial \varepsilon_{i j}}, \quad \varphi=-\frac{\partial W_{\mathrm{s}}}{\partial p}
$$

For the sake of simplicity, we adopt a reference configuration free of stress and pore pressure. In linear poroelasticity, $W_{\mathrm{s}}$ is then a quadratic form of its arguments $\varepsilon_{i j}$ and $p$. Restricting to an isotropic porous solid and letting again $\varepsilon$ be the volumetric strain $\varepsilon_{k k}$, from (18) we finally get

$$
\begin{aligned}
\sigma_{i j} & =(K-2 G / 3) \varepsilon \delta_{i j}+2 G \varepsilon_{i j}-b p \delta_{i j} \\
\varphi & =b \varepsilon+p / N
\end{aligned}
$$

where properties $K$ and $G$ are the bulk modulus and the shear modulus of the empty porous solid, that is, with a zero pore pressure $(p=0)$; poroelastic properties $b$ and $N$ are, respectively, the Biot coefficient [2] and the Biot modulus [14]. These macroscopic poroelastic properties are linked to the bulk modulus $k_{\mathrm{S}}$ of the solid matrix forming the solid part of the porous solid according to the well-known relations (see, for instance, [15])

$$
b=1-K / k_{\mathrm{s}}, \quad 1 / N=\left(b-\phi_{0}\right) / k_{\mathrm{s}}
$$

Constitutive equation (19) may be formally rewritten in the form

$$
\sigma_{i j}^{\prime \prime}=(K-2 G / 3) \varepsilon \delta_{i j}+2 G \varepsilon_{i j}
$$


where

$$
\sigma_{i j}^{\prime \prime}=\sigma_{i j}+b p \delta_{i j}
$$

may be coined as Biot's effective stress since constitutive equation (22) looks the same as the one related to an ordinary elastic solid with $K$ and $G$ as elastic properties. If the solid matrix is incompressible ( $\varepsilon_{\mathrm{S}}=0$ in (6)), $k_{\mathrm{s}}$ goes to infinity so that $b$ goes to 1 and $1 / N$ goes to zero in (21). As expected constitutive equation (20) then reduces to relation (7) expressing the incompressibility of the solid matrix while Biot's effective stress $\sigma_{i j}^{\prime \prime}$ reduces to the celebrated Terzaghi's effective stress $\sigma_{i j}^{\prime}$ of soil mechanics defined by

$$
\sigma_{i j}^{\prime}=\sigma_{i j}+p \delta_{i j}
$$

Constitutive equations (19) and (20) can be easily extended to thermoporoelasticity in the form

$$
\begin{aligned}
\sigma_{i j} & =(K-2 G / 3) \varepsilon \delta_{i j}+2 G \varepsilon_{i j}-b p \delta_{i j}-3 a K \theta \delta_{i j} \\
\varphi & =b \varepsilon+p / N+3 a_{\varphi} \theta
\end{aligned}
$$

where $3 a$ and $3 a_{\varphi}$ are the volumetric thermal dilation coefficients related, respectively, to the empty porous solid and to the porous volume. It can be shown (see, for instance, [15])

$$
a=\alpha_{\mathrm{s}}, \quad a_{\varphi}=\alpha_{\mathrm{s}}\left(b-\phi_{0}\right)
$$

where $3 \alpha_{\mathrm{s}}$ is the volumetric thermal dilation coefficient of the solid matrix.

\subsection{Saturated poroplasticity}

Saturated poroelasticity can be easily extended to saturated poroplasticity by considering the plastic deformation $\varepsilon_{i j}^{\mathrm{p}}$ and the plastic change of porosity $\varphi^{\mathrm{p}}$. For the sake of simplicity, we will restrict to ideal poroplasticity, ignoring possible hardening effects which can be easily addressed in the general framework proposed here (for hardening effects and the associated frozen energy see, for instance, $[15,30])$. Accordingly, the free energy $F$ has for arguments the elastic deformation $\varepsilon_{i j}-\varepsilon_{i j}^{\mathrm{p}}$ and the elastic change of porosity $\varphi-\varphi^{\mathrm{p}}$. We write

$$
F_{\mathrm{S}}=F_{\mathrm{S}}\left(\varepsilon_{i j}-\varepsilon_{i j}^{\mathrm{p}}, \varphi-\varphi^{\mathrm{p}}\right)
$$

Substitution of (28) in (13) and use of (15) provide

$$
\sigma_{i j} \mathrm{~d} \varepsilon_{i j}^{\mathrm{p}}+p \mathrm{~d} \varphi^{\mathrm{p}} \geqslant 0
$$

In the general case, $\varepsilon_{i j}^{\mathrm{p}}$ and $\varphi^{\mathrm{p}}$ are independent variables and will be governed by separate flow rules so that the plastic yield function $f$ will have $\sigma_{i j}$ and $p$ as separate arguments. Restricting to associated flow rules for the sake of simplicity, we write

$$
\mathrm{d} \varepsilon_{i j}^{\mathrm{p}}=\mathrm{d} \lambda \frac{\partial f\left(\sigma_{i j}, p\right)}{\partial \sigma_{i j}}, \quad \mathrm{~d} \varphi^{\mathrm{p}}=\mathrm{d} \lambda \frac{\partial f\left(\sigma_{i j}, p\right)}{\partial p}
$$

where $\mathrm{d} \lambda$ stands for the plastic multiplier. 
From (6), irrespective of saturated or unsaturated conditions, we get the plastic volume balance in the form

$$
\mathrm{d} \varepsilon_{k k}^{\mathrm{p}}=\mathrm{d} \varepsilon^{\mathrm{p}}=\left(1-\phi_{0}\right) \mathrm{d} \varepsilon_{\mathrm{s}}^{\mathrm{p}}+\mathrm{d} \varphi^{\mathrm{p}}
$$

In the particular case of a plastically incompressible solid matrix, that is $d \varepsilon_{\mathrm{s}}^{\mathrm{p}}=0$, the previous volume balance reduces to

$$
\mathrm{d} \varepsilon_{k k}^{\mathrm{p}}=\mathrm{d} \varphi^{\mathrm{p}}
$$

Substitution of (32) in (29) provides

$$
\sigma_{i j}^{\prime} \mathrm{d} \varepsilon_{i j}^{\mathrm{p}} \geqslant 0
$$

where $\sigma_{i j}^{\prime}$ is Terzaghi's effective stress we defined in (24). According to (30) and (33), the plastic yield function $f$ has then to take the specific form $f=f\left(\sigma_{i j}^{\prime}\right)$. Since the solid matrix forming the solid part of the porous solid may be simultaneously elastically compressible (that is, $k_{\mathrm{s}} \neq \infty, b \neq 1$ ) and plastically incompressible (that is, (32) holds), Biot's (elastic) effective stress, $\sigma_{i j}^{\prime \prime}=\sigma_{i j}+b p \delta_{i j}$, and Terzaghi's (plastic) effective stress, $\sigma_{i j}^{\prime}$, may be simultaneously relevant for the same porous material without necessarily coinciding.

\section{UNSATURATED POROELASTOPLASTICITY FOR DISCONNECTED NETWORKS}

\subsection{Lagrangian saturation}

We now consider the case where the porous volume is formed of two disconnected porous networks referred to by index $J=1,2$. By disconnected we mean that the porous networks are not communicating so that each porous network $J$ remains fully saturated by the same fluid $J$ in all the subsequent evolutions of the porous solid. The overall porosity is $\phi_{0}$ in the reference initial configuration, and $\phi$ in the current configuration. The overall porosity $\phi$ can be split into two partial (Lagrangian) porosities, $\phi_{1}$ and $\phi_{2}$, according to

$$
\phi=\phi_{1}+\phi_{2}
$$

Similarly to the overall porosity, the current partial porosity $\phi_{J}$ may be written in the form

$$
\phi_{J}=\phi_{0} S_{J}+\varphi_{J}, \quad S_{1}+S_{2}=1
$$

Substituting the decomposition (35) of $\phi_{J}$ in (34), and the result of this substitution in (1), the overall change $\varphi$ of the initial porosity $\phi_{0}$ which can be due only to the deformation, is now split into the contributions $\varphi_{1}$ and $\varphi_{2}$ related to each network according to

$$
\varphi=\phi-\phi_{0}=\varphi_{1}+\varphi_{2}
$$

Since $\varphi_{J}$ finally represents in (35) the contribution to the partial porosity $\phi_{J}$ due only to the deformation of the porous network $J$, the term $\phi_{0} S_{J}$ is the current partial saturation related to the fluid $J$ prior to any deformation. In this section, the two porous networks are disconnected so that each fluid occupies a part of the porous volume which is always delimited by the same internal solid walls. As a result $S_{J}$ will remain constant, resulting in $\mathrm{d} S_{J}=0$, and the variation of the 
partial Lagrangian porosity $\phi_{J}$ is only due to the deformation of the porous network $J$, resulting in $\mathrm{d} \phi_{J}=\mathrm{d} \varphi_{J}$. As explored in the next section, this will be no longer true in the case of connected porous networks since each fluid may then invade the porous solid, or recede from it so that the fluids do not remain in contact with the same internal solid walls throughout the evolutions of the porous solid.

The current partial saturation $s_{J}$ is more usually defined with regard to the deformed current configuration according to

$$
\phi_{J}=\phi s_{J}, \quad s_{1}+s_{2}=1
$$

In contrast to $S_{J}$, even in the case of disconnected porous networks, the saturation $s_{J}$ will not remain constant because of the deformation of the porous volume. Saturation $s_{J}$ stands for the current volume fraction of fluid $J$ relatively to the deformed current porous volume $\phi \times \mathrm{d} \Omega_{0}$ so that it can be coined as the Eulerian saturation. In contrast $S_{J}$ is always related to the undeformed initial porous volume $\phi_{0} \times \mathrm{d} \Omega_{0}$ and it can be coined as the Lagrangian saturation. As for the porosity in saturated conditions, the use of the Lagrangian saturation $S_{J}$, instead of the Eulerian saturation $s_{J}$, will shortly turn out to be more efficient, even in case of infinitesimal strains.

\subsection{Unsaturated thermoporoelasticity}

With definition (35), energy balance (14) extends in the form

$$
\sigma_{i j} \mathrm{~d} \varepsilon_{i j}+p_{1} \mathrm{~d} \varphi_{1}+p_{2} \mathrm{~d} \varphi_{2}-\mathrm{d} F_{\mathrm{s}}=0
$$

and, analogously to (15), we have

$$
\sigma_{i j}=\frac{\partial F_{\mathrm{s}}}{\partial \varepsilon_{i j}}, \quad p_{1}=\frac{\partial F_{\mathrm{s}}}{\partial \varphi_{1}}, \quad p_{2}=\frac{\partial F_{\mathrm{s}}}{\partial \varphi_{2}}
$$

Accordingly, constitutive equations (25) and (26) extend in the form

$$
\begin{aligned}
\sigma_{i j} & =(K-2 G / 3) \varepsilon \delta_{i j}+2 G \varepsilon_{i j}-b_{1} p_{1} \delta_{i j}-b_{2} p_{2} \delta_{i j}-3 a K \theta \delta_{i j} \\
\varphi_{1} & =b_{1} \varepsilon+p_{1} / N_{11}+p_{2} / N_{12}+3 a_{1} \theta \\
\varphi_{2} & =b_{2} \varepsilon+p_{1} / N_{12}+p_{2} / N_{22}+3 a_{2} \theta
\end{aligned}
$$

When $p_{1}=p_{2}$ we must retrieve the saturated case so that we get

$$
b=b_{1}+b_{2}, \quad 1 / N_{11}+2 / N_{12}+1 / N_{22}=1 / N, \quad a_{1}+a_{2}=a_{\varphi}
$$

where $b, N$ and $a_{\varphi}$ are the thermoporoelastic properties related to the porous solid previously introduced and, thereby, still subjected to relations (21) and (27). It can be further shown (see [14] and Appendix A) that

$$
1 / N_{J J}+1 / N_{12}=\left(b_{J}-\phi_{0} S_{J}\right) / k_{\mathrm{s}}, \quad a_{J}=\alpha_{\mathrm{s}}\left(b_{J}-\phi_{0} S_{J}\right)
$$

\subsection{Effective pore pressure and the iso-deformation assumption}

The first relation of (43) allows us to write

$$
b_{1}=b \chi, \quad b_{2}=b(1-\chi)
$$


where $\chi$ is a constant depending on the fixed Lagrangian saturation $S_{1}$. Using relations (21), (43)-(45) in (40), we get

$$
\begin{aligned}
\sigma_{i j} & =(K-2 G / 3) \varepsilon \delta_{i j}+2 G \varepsilon_{i j}-b\left[\chi p_{1}+(1-\chi) p_{2}\right] \delta_{i j}-3 a K \theta \delta_{i j} \\
\varphi & =\varphi_{1}+\varphi_{2}=b \varepsilon+\left\{\left[b \chi-\phi_{0} S_{1}\right] p_{1}+\left[b(1-\chi)-\phi_{0} S_{2}\right] p_{2}\right\} /\left[N\left(b-\phi_{0}\right)\right]+3 a_{\varphi} \theta
\end{aligned}
$$

If we replace the pore pressure $p$ by $\chi p_{1}+(1-\chi) p_{2}$ in the constitutive equation (25) related to the saturated case, we get (46). On this only basis $\chi p_{1}+(1-\chi) p_{2}$ is often claimed to act as an effective pore pressure as the pore pressure $p$ does in the saturated case. Unfortunately, the substitution of $p=\chi p_{1}+(1-\chi) p_{2}$ in the second constitutive equation (26) does not provide (47). In fact, with no further information, relations (43) and (44) capitalize all that can be actually derived in the unsaturated isotropic poroelastic case.

A useful assumption, sometimes implicitly made [8,15], or explicitly explored [31], may be the iso-deformation of the two porous networks. This assumption states that the porous volume occupied by each of the two fluids deforms the same whenever they are subjected to no pressure and temperature variations. This assumption amounts to writing

$$
p_{1}=p_{2}=\theta=0: \varphi_{1} /\left(\phi_{0} S_{1}\right)=\varphi_{2} /\left(\phi_{0} S_{2}\right)
$$

Substitution of (48) in (41) and (42) provides

$$
b_{1} / S_{1}=b_{2} / S_{2}
$$

Substituting (49) in the first of relations (43), we get the identification

$$
b_{J}=b S_{J}, \quad \chi=S_{1}
$$

Because of relations (44) and (50), it can be easily shown that the porous volumes related to each fluid keep deforming the same provided they are subjected to the same pressure and temperature variations (see Appendix A). Using (50) in (46) and (47), constitutive equations (25) and (26) of saturated thermoporoelasticity are now both retrieved, provided that the effective pore pressure $p$ is identified with $S_{1} p_{1}+S_{2} p_{2}$. Although the inappropriate use of the Eulerian saturations $s_{J}$ defined in (37) may unfortunately lead to the opposite conclusion (see [15] and Appendix B), the pressure $S_{1} p_{1}+S_{2} p_{2}$ may play the role of an effective pore pressure if and only if the iso-deformation assumption (48) holds. This conclusion has been already reached in [31] from micromechanical arguments. The iso-deformation assumption (48) holds in limited situations. For instance it holds in the dilute approximation for spherical pores. Unfortunately, as soon as the dilute approximation is no more relevant, it can be shown that (50) does not apply because of the coupling between the deformation of pores having different sizes. Accordingly the use of $S_{1} p_{1}+S_{2} p_{2}$ as an effective pore pressure remains questionable for most of natural materials.

\subsection{Unsaturated poroplasticity}

Analogously to the saturated case, in addition to the plastic deformation $\varepsilon_{i j}^{\mathrm{p}}$, unsaturated poroplasticity consists of considering a plastic change in partial porosity $\varphi_{J}^{\mathrm{p}}$ relatively to each porous network so that (29) and (30) extend in the form

$$
\sigma_{i j} \mathrm{~d} \varepsilon_{i j}^{\mathrm{p}}+p_{1} \mathrm{~d} \varphi_{1}^{\mathrm{p}}+p_{2} \mathrm{~d} \varphi_{2}^{\mathrm{p}} \geqslant 0
$$


and

$$
\mathrm{d} \varepsilon_{i j}^{\mathrm{p}}=\mathrm{d} \lambda \frac{\partial f\left(\sigma_{i j}, p_{1}, p_{2}, S_{1}\right)}{\partial \sigma_{i j}}, \quad \mathrm{~d} \varphi_{J}^{\mathrm{p}}=\mathrm{d} \lambda \frac{\partial f\left(\sigma_{i j}, p_{1}, p_{2}, S_{1}\right)}{\partial p_{J}}
$$

The overall plastic porosity is the sum of the partial plastic porosity and we write

$$
\mathrm{d} \varphi^{\mathrm{p}}=\mathrm{d} \varphi_{1}^{\mathrm{p}}+\mathrm{d} \varphi_{2}^{\mathrm{p}}
$$

As a consequence, the general relation (32) capturing the plastic incompressibility of the solid matrix, i.e. $d \varepsilon_{\mathrm{s}}^{\mathrm{p}}=0$, is now expressed in the form

$$
\mathrm{d} \varepsilon_{k k}^{\mathrm{p}}=\mathrm{d} \varphi_{1}^{\mathrm{p}}+\mathrm{d} \varphi_{2}^{\mathrm{p}}
$$

According to (52) and (54), the plastic yield function $f$ has then to take the specific form

$$
f=f\left(\sigma_{i j}+p_{1} \delta_{i j}, \sigma_{i j}+p_{2} \delta_{i j}\right)
$$

involving the two 'effective stresses' $\sigma_{i j}+p_{1} \delta_{i j}$ and $\sigma_{i j}+p_{2} \delta_{i j}$. In contrast to the saturated case, even though the plastic incompressibility condition (54) holds as for soils, no unique plastic effective stress may thereby be unquestionably identified.

Relation (53) suggests the existence of a material constant $\gamma$ such that

$$
\mathrm{d} \varphi_{1}^{\mathrm{p}}=\gamma \mathrm{d} \varphi^{\mathrm{p}}, \quad \mathrm{d} \varphi_{2}^{\mathrm{p}}=(1-\gamma) \mathrm{d} \varphi^{\mathrm{p}}
$$

depending, in particular, on the fixed Lagrangian saturation $S_{1}$. However, it must be pointed out that, in contrast to the material constant $\chi$ defined by (45) and whose existence is ensured by the linearity of the poroelastic behaviour, there is no reason that such a material constant $\gamma$ does exist whatever the material considered. If a function $\gamma$ may always be formally introduced through relation (56), in the general case its current value depends on the current loading $\left(\sigma_{i j}, p_{J}\right)$ through the flow rule. The introduction of $\gamma$ will then present no actual interest. Relation (56), where $\gamma$ is a material constant depending on $S_{1}$, remains an assumption. Substituting then (56) in (51), we get

$$
\sigma_{i j} \mathrm{~d} \varepsilon_{i j}^{\mathrm{p}}+\left[\gamma p_{1}+(1-\gamma) p_{2}\right] \mathrm{d} \varphi^{\mathrm{p}} \geqslant 0
$$

The parallel between (57) and (29) shows that $\gamma p_{1}+(1-\gamma) p_{2}$ plays the role of an effective pressure now regarding the poroplastic behaviour. It is then tempting to extend the iso-deformation assumption (48) to the plastic case in the form

$$
\gamma=S_{1}, \quad\left(\mathrm{~d} \varphi_{J}^{\mathrm{p}}=S_{J} \mathrm{~d} \varphi^{\mathrm{p}}\right)
$$

so that $\gamma p_{1}+(1-\gamma) p_{2}$ is identified with the averaged pore pressure $S_{1} p_{1}+S_{2} p_{2}$.

Combining the plastic incompressibility condition (32), with the plastic iso-deformation assumption (58), (57) reduces to (33) provided that $\sigma_{i j}^{\prime}$ is now identified with

$$
\sigma_{i j}^{\prime}=\sigma_{i j}+\left(S_{1} p_{1}+S_{2} p_{2}\right) \delta_{i j}
$$

When letting $S_{1} p_{1}+S_{2} p_{2}$ play the role of an effective pore pressure, Terzaghi's definition (24) of the effective stress with regard to the plastic behaviour is then ultimately recovered. However, the plastic iso-deformation assumption (58) may hold only in limited situations, similar to the one evoked when discussing the validity of the iso-deformation assumption (48). 


\section{UNSATURATED POROELASTOPLASTICITY FOR CONNECTED NETWORKS}

We now want to examine the more general case of connected porous networks. In this case, the porous volume is partially saturated by a non-wetting fluid, referred to by index nwF, while the remaining porous space is filled by a wetting fluid, referred to by wF. A familiar situation encountered in many applications is the case where the wetting fluid is liquid water while the non-wetting fluid is air. More generally they may be a gas or a liquid, pure fluids or mixtures. The saturating fluids are only assumed to be immiscible and to remain connected. For water-infiltrated granular materials like soils, this amounts to assuming that the liquid water content trapped in the intergranular menisci in the air-dominated part of the porous volume is negligible and whose effects can be accounted for through more specific approaches [32]. As for disconnected porous networks, current partial (Lagrangian) porosities $\phi_{\mathrm{wF}}$ and $\phi_{\mathrm{nwF}}$ can then be associated with the wetting and the non-wetting fluid, respectively, and (11) extends in the form

$$
\sigma_{i j} \mathrm{~d} \varepsilon_{i j}+p_{\mathrm{wF}} \mathrm{d} \phi_{\mathrm{wF}}+p_{\mathrm{nwF}} \mathrm{d} \phi_{\mathrm{nwF}}-\mathrm{d} F \geqslant 0
$$

\subsection{Solid-fluid interface energy and the Lagrangian saturation}

Similarly to (35), we now write

$$
\phi_{\mathrm{wF}}=\phi_{0} S_{\mathrm{wF}}+\varphi_{\mathrm{wF}}, \quad \phi_{\mathrm{nwF}}=\phi_{0} S_{\mathrm{nwF}}+\varphi_{\mathrm{nwF}}, \quad S_{\mathrm{wF}}+S_{\mathrm{nwF}}=1
$$

However for connected porous networks, the fluids can now invade the porous solid, or recede from it, so that they are no more in contact with the same internal solid walls delimiting the porous network. As a result the Lagrangian saturations $S_{\mathrm{wF}}$ and $S_{\mathrm{nwF}}$ are no more constant. The reference state may be conveniently chosen as fully saturated by one of the two fluids, the wetting fluid, for instance, so that we have $S_{\mathrm{wF}}=1$ in the reference state. The associated initial pore pressure is generally equal to the atmospheric pressure taken as the zero pressure reference. If now we force the non-wetting fluid to enter the porous solid by increasing the pressure difference $p_{\mathrm{nwF}}-p_{\mathrm{wF}}$, the non-wetting fluid saturation $S_{\mathrm{nwF}}$ will increase. The wetting fluid will be simultaneously drained and $S_{\mathrm{wF}}$ will decrease according to the last of relations (61). During the drainage the porous networks simultaneously deform. As previously mentioned, owing to its very definition the Lagrangian saturation $S_{\mathrm{wF}}$ still refers the currently wetted zone to the undeformed reference configuration. Actually, $S_{\mathrm{wF}} \phi_{0} \times \mathrm{d} \Omega_{0}$ is the porous volume delimited in the undeformed reference configuration by the internal solid walls that will remain wetted in the current deformed configuration. In the current deformed configuration the same wetted walls delimit the deformed volume $\left(S_{\mathrm{wF}} \phi_{0}+\varphi_{\mathrm{wF}}\right) \times \mathrm{d} \Omega_{0}$, the volume $\varphi_{\mathrm{wF}} \times \mathrm{d} \Omega_{0}$ capturing the current change of the initial porous volume $S_{\mathrm{wF}} \phi_{0} \times \mathrm{d} \Omega_{0}$ due to the deformation.

Substituting (61) in (60), we get

$$
\left(\sigma_{i j} \mathrm{~d} \varepsilon_{i j}+p_{\mathrm{wF}} \mathrm{d} \varphi_{\mathrm{wF}}+p_{\mathrm{nwF}} \mathrm{d} \varphi_{\mathrm{nwF}}\right)-\phi_{0}\left(p_{\mathrm{nwF}}-p_{\mathrm{wF}}\right) \mathrm{d} S_{\mathrm{wF}}-\mathrm{d} F \geqslant 0
$$

Owing to the previous analysis the first term in parenthesis is still identified with the strain work supplied to the porous solid between time $t$ and $t+\mathrm{d} t$. The second term identifies with the energy required to create new inner interfaces between the fluids and the solid matrix, provided that we only consider the energy needed to make the interface between the two fluids move and we neglect the energy needed to deform it. According to (62), and in contrast to the case of disconnected networks, the free energy $F$ does change not only because of the deformation of the porous 
solid, but also because of the change in the energy associated with the fluid-solid interface resulting from the variation of $S_{\mathrm{wF}}$. As a result the identification (12) of $F$ with the free energy $F_{\mathrm{S}}$ of the solid matrix no longer holds. Accounting for the interface energy changes, but ignoring the influence of the temperature for the sake of simplicity, we write

$$
F=F_{\mathrm{s}}\left(\varepsilon_{i j}, \varphi_{\mathrm{wF}}, \varphi_{\mathrm{nwF}}, \chi\right)+\phi_{0} U\left(S_{\mathrm{wF}}\right)
$$

The first term $F_{\mathrm{s}}\left(\varepsilon_{i j}, \varphi_{\mathrm{wF}}, \varphi_{\mathrm{nwF}}, \chi\right)$ still stands for the free energy associated with the solid matrix. The current value of the free energy depends on the current partition of the porous volume between the wetted part and the non-wetted part. This is captured through the dependence of $F$ on the variable $\chi$, which itself is a function of the current saturation $S_{\mathrm{wF}}$ and whose notation refers to relation (45), anticipating relation (69). The term $\phi_{0} U$ accounts for the current value of the energy of the interface between the solid and the fluids. Similar to the assumption implicitly made in the saturated case, the effects of the deformation of the internal walls of the porous solid on the interface energy are assumed to be negligible, so that only the Lagrangian saturation $S_{\mathrm{wF}}$ has been retained as argument of the interface energy $U$ per unit of porous volume $\phi_{0} \times \mathrm{d} \Omega_{0}$.

\subsection{Unsaturated poroelasticity and capillary hysteresis}

In case of a poroelastic solid, analogously to state equations (39) we now write

$$
\sigma_{i j}=\frac{\partial F_{\mathrm{s}}}{\partial \varepsilon_{i j}}, \quad p_{\mathrm{wF}}=\frac{\partial F_{\mathrm{s}}}{\partial \varphi_{\mathrm{wF}}}, \quad p_{\mathrm{nwF}}=\frac{\partial F_{\mathrm{s}}}{\partial \varphi_{\mathrm{nwF}}}
$$

Following the previous analysis we made for the case of disconnected porous networks and restricting to isothermal conditions, (64) allows us to extend (40)-(42) in the form

$$
\begin{aligned}
\sigma_{i j} & =(K-2 G / 3) \varepsilon \delta_{i j}+2 G \varepsilon_{i j}-b_{\mathrm{wF}} p_{\mathrm{wF}} \delta_{i j}-b_{\mathrm{nwF}} p_{\mathrm{nwF}} \delta_{i j} \\
\varphi_{\mathrm{wF}} & =b_{\mathrm{wF}} \varepsilon+p_{\mathrm{wF}} / N_{\mathrm{wF}-\mathrm{wF}}+p_{\mathrm{wF}} / N_{\mathrm{wF}-\mathrm{nwF}} \\
\varphi_{\mathrm{nwF}} & =b_{\mathrm{nwF}} \varepsilon+p_{\mathrm{wF}} / N_{\mathrm{wF}-\mathrm{nwF}}+p_{\mathrm{nwF}} / N_{\mathrm{nwF}-\mathrm{nwF}}
\end{aligned}
$$

Substitution of (63) and (64) in (62) provides

$$
-\phi_{0}\left(p_{\mathrm{nwF}}-p_{\mathrm{wF}}\right) \mathrm{d} S_{\mathrm{wF}}-\phi_{0} \mathrm{~d} U-\frac{\partial F_{\mathrm{s}}}{\partial \chi} \mathrm{d} \chi \geqslant 0
$$

In linear poroelasticity $F_{\mathrm{S}}$ is a quadratic function of its arguments, $\varepsilon_{i j}, \varphi_{\mathrm{nwF}}$, and $\varphi_{\mathrm{nwF}}$. The coefficients of this form are function of $\chi$ as, for instance, the coefficients $b_{\mathrm{wF}}$ and $b_{\mathrm{nwF}}$ which, similar to $b_{1}$ and $b_{2}$ in (45), have to satisfy

$$
b_{\mathrm{wF}}=b \chi\left(S_{\mathrm{wF}}\right), \quad b_{\mathrm{nwF}}=b\left[1-\chi\left(S_{\mathrm{wF}}\right)\right]
$$

Accordingly, $\partial F_{\mathrm{s}} / \partial \chi$ is a second-order term since it only involves quadratic terms such as $\varepsilon_{i j} \varepsilon_{k l}$, $\varepsilon_{i j} \varphi_{\mathrm{wF} \text { or nwF }}$, or $\varphi_{\mathrm{wF}} \varphi_{\mathrm{nwF}}$ and that $\varepsilon_{i j}, \varphi_{\mathrm{nwF}}, \varphi_{\mathrm{nwF}}$ are infinitesimal quantities. As a result, neglecting $\partial F_{\mathrm{s}} / \partial \chi$ in (68) we get

$$
-\left(p_{\mathrm{nwF}}-p_{\mathrm{wF}}\right) \mathrm{d} S_{\mathrm{wF}}-\mathrm{d} U \geqslant 0
$$


Inequality (70) captures the dissipation associated with capillary hysteresis. In the absence of significant capillary hysteresis (70) becomes an equality and we may write

$$
p_{\mathrm{nwF}}-p_{\mathrm{wF}}=-\frac{\mathrm{d} U\left(S_{\mathrm{wF}}\right)}{\mathrm{d} S_{\mathrm{wF}}}
$$

It results in a one-to-one relationship between the capillary pressure $p_{\mathrm{nwF}}-p_{\mathrm{wF}}$ and the saturation $S_{\mathrm{wF}}$ which can be retrieved at the pore scale [33]. In contrast to (71), capillary hysteresis implies that the relation linking $p_{\mathrm{nwF}}-p_{\mathrm{wF}}$ and $S_{\mathrm{wF}}$ will not be the same for a drainage process $\left(S_{\mathrm{wF}} \downarrow\right)$ than for an imbibition process $\left(S_{\mathrm{wF}} \uparrow\right)$. Thereby, for the same value of the capillary pressure $p_{\mathrm{nwF}}-p_{\mathrm{wF}}$, the $\chi$ variable, and accordingly the poroelastic properties involved in (65)-(67), will not have the same values in a drainage process and in an imbibition process. As a result, in a drainage-imbibition test a capillary-induced irreversibility of the strain history will be observed, even though the porous solid is elastic. Capillary hysteresis can be addressed in the formalism of plasticity $[24,34,35]$.

\subsection{Unsaturated poroplasticity for connected porous networks}

The poroplastic analysis we did for disconnected porous networks extends to the case of connected porous networks in a similar way, by replacing $\varepsilon_{i j}, \varphi_{\mathrm{wF}}$, and $\varphi_{\mathrm{nwF}}$ in (65)-(67) by, respectively, $\varepsilon_{i j}-\varepsilon_{i j}^{\mathrm{p}}, \varphi_{\mathrm{wF}}-\varphi_{\mathrm{wF}}^{\mathrm{p}}, \varphi_{\mathrm{nwF}}-\varphi_{\mathrm{nwF}}^{\mathrm{p}}$. We now write the plastic dissipation in the form

$$
\sigma_{i j} \mathrm{~d} \varepsilon_{i j}^{\mathrm{p}}+p_{\mathrm{wF}} \mathrm{d} \varphi_{\mathrm{wF}}^{\mathrm{p}}+p_{\mathrm{nwF}} \mathrm{d} \varphi_{\mathrm{nwF}}^{\mathrm{p}} \geqslant 0
$$

with the related plastic flow rule

$$
\mathrm{d} \varepsilon_{i j}^{\mathrm{p}}=\mathrm{d} \lambda \frac{\partial f}{\partial \sigma_{i j}}, \quad \mathrm{~d} \varphi_{\mathrm{wF}}^{\mathrm{p}}=\mathrm{d} \lambda \frac{\partial f}{\partial p_{\mathrm{wF}}}, \quad \mathrm{d} \varphi_{\mathrm{nwF}}^{\mathrm{p}}=\mathrm{d} \lambda \frac{\partial f}{\partial p_{\mathrm{nwF}}}
$$

where $f$ is the plastic yield function:

$$
f=f\left(\sigma_{i j}, p_{\mathrm{wF}}, p_{\mathrm{nwF}}, S_{\mathrm{wF}}\right)
$$

In contrast to $S_{1}$ which remained constant in (52), the Lagrangian saturation $S_{\mathrm{wF}}$ is no more a constant in (74). As a result, even in the absence of any capillary hysteresis, the history of the plastic deformation and of the plastic change of porosity will depend on the chronology of the saturation history $S_{\mathrm{wF}}$ relatively to the chronology of the plastic loading $\sigma_{i j}, p_{\mathrm{wF}}$, and $p_{\mathrm{nwF}}$.

Plastic flow rule (73) is quite general. In various domains as in soil mechanics, the solid matrix is formed of grains and may be considered as plastically incompressible, that is $\mathrm{d} \varepsilon_{\mathrm{s}}^{\mathrm{p}}=0$. Updating the notations of (54) we then write

$$
\mathrm{d} \varepsilon_{k k}^{\mathrm{p}}=\mathrm{d} \varphi^{\mathrm{p}}=\mathrm{d} \varphi_{\mathrm{wF}}^{\mathrm{p}}+\mathrm{d} \varphi_{\mathrm{nwF}}^{\mathrm{p}}
$$

Substitution of (75) in (72) provides

$$
\left(\sigma_{i j}+p_{\mathrm{nwF}} \delta_{i j}\right) \mathrm{d} \varepsilon_{i j}^{\mathrm{p}}-\left(p_{\mathrm{nwF}}-p_{\mathrm{wF}}\right) \mathrm{d} \varphi_{\mathrm{wF}}^{\mathrm{p}} \geqslant 0
$$

so that the flow rule becomes

$$
\mathrm{d} \varepsilon_{i j}^{\mathrm{p}}=\mathrm{d} \lambda \frac{\partial f}{\partial\left(\sigma_{i j}+p_{\mathrm{nwF}} \delta_{i j}\right)}, \quad \mathrm{d} \varphi_{\mathrm{wF}}^{\mathrm{p}}=-\mathrm{d} \lambda \frac{\partial f}{\partial\left(p_{\mathrm{nwF}}-p_{\mathrm{wF}}\right)}
$$


where the plastic yield function (74) is expressed in the form

$$
f=f\left(\sigma_{i j}+p_{\mathrm{nwF}} \delta_{i j}, p_{\mathrm{nwF}}-p_{\mathrm{wF}}, S_{\mathrm{wF}}\right)
$$

We retrieve here the need for defining two effective stresses, even though the solid matrix is plastically incompressible. The analysis which led to (55) for disconnected porous networks would have here lead to the choice of the two effective stresses $\sigma_{i j}+p_{\mathrm{wF}} \delta_{i j}$ and $\sigma_{i j}+p_{\mathrm{nwF}} \delta_{i j}$. This choice of one stress variable relative to the voids filled by the wetting fluid, and one distinct stress variable relative to the voids filled by the non-wetting fluid, has been already suggested in [35]. In conformity with usual practice in soil mechanics [27], the alternative choice retained in (77)-(78) consists of the so-called net stress $\sigma_{i j}+p_{\mathrm{nwF}} \delta_{i j}$ associated with the plastic strain increment $\mathrm{d} \varepsilon_{i j}^{\mathrm{p}}$, and of the pressure difference $p_{\mathrm{nwF}}-p_{\mathrm{wF}}$ associated with the plastic increment of partial porosity change $\mathrm{d} \varphi \varphi_{\mathrm{wF}}^{\mathrm{p}}$. However, this alternative choice may lead to some confusion with regard to the twofold role played by the pressure difference $p_{\mathrm{nwF}}-p_{\mathrm{wF}}$. In addition to be the loading plastic parameter associated with $\mathrm{d} \varphi_{\mathrm{wF}}^{\mathrm{p}}$, the pressure difference $p_{\mathrm{nwF}}-p_{\mathrm{wF}}$ also stands for the capillary pressure which, according to (71), is the energy conjugate variable of saturation $S_{\mathrm{wF}}$ with regard to interface energy $U$.

The general plastic flow rule (73) accounts only for the irreversible change of porosity due to the current plastic yielding. It does not account for the irreversible porosity change previously undergone by the infinitesimal porous volume newly invaded (or left) by the fluid considered. This contribution can be roughly accounted for by modifying (73) in the form

$$
\mathrm{d} \varphi_{\mathrm{wF}}^{\mathrm{p}}=\mathrm{d} \lambda \frac{\partial f}{\partial p_{\mathrm{wF}}}+\left(\varphi_{\mathrm{nwF}}^{\mathrm{p}}-\varphi_{\mathrm{wF}}^{\mathrm{p}}\right) \mathrm{d} S_{\mathrm{wF}}, \quad \mathrm{d} \varphi_{\mathrm{nwF}}^{\mathrm{p}}=\mathrm{d} \lambda \frac{\partial f}{\partial p_{\mathrm{nwF}}}-\left(\varphi_{\mathrm{nwF}}^{\mathrm{p}}-\varphi_{\mathrm{wF}}^{\mathrm{p}}\right) \mathrm{d} S_{\mathrm{wF}}
$$

Incremental constitutive equations (79) guarantee that the overall plastic porosity change $\mathrm{d} \varphi^{\mathrm{p}}$ is actually only due to the current plastic yielding according to

$$
\mathrm{d} \varphi^{\mathrm{p}}=\mathrm{d} \varphi_{\mathrm{wF}}^{\mathrm{p}}+\mathrm{d} \varphi_{\mathrm{nwF}}^{\mathrm{p}}=\mathrm{d} \lambda\left(\frac{\partial f}{\partial p_{\mathrm{wF}}}+\frac{\partial f}{\partial p_{\mathrm{nwF}}}\right)
$$

Incremental constitutive equations (79) also guarantee that the internal solid walls of the porous solid keep the memory of the irreversible changes of partial porosities previously undergone, whatever the current state of saturation is. For instance, let us consider a complete drainage process leaving the porous solid fully saturated by the non-wetting fluid, that is $S_{\mathrm{wF}}=0$, and resulting in a final plastic porosity $\varphi^{\mathrm{p}}=\varphi_{\mathrm{nwF}}^{\mathrm{p}}$. If later on the porous solid is subjected to an imbibition process $\left(S_{\mathrm{wF}} \uparrow\right)$ that is purely elastic $(\mathrm{d} \lambda=0)$, incremental constitutive equations (79) guarantee that, during the imbibition, the porous solid will keep the memory of the plastic change $\varphi^{\mathrm{p}}=\varphi_{\mathrm{nwF}}^{\mathrm{p}}$ previously undergone during the former drainage. In short, Lagrangian partial plastic porosity changes are associated with the irreversible deformation undergone by the solid internal walls delimiting the volumes currently occupied by the saturating fluids. They are not associated with the saturating fluids themselves.

\section{SUMMARY AND CONCLUSIONS}

Through an energy approach we revisited the constitutive equations of unsaturated porous solids at the light of a Lagrangian saturation concept. The Lagrangian saturation is the cornerstone to 
distinguish the energy required for the invasion of the porous network by the non-wetting fluid from the energy required for the simultaneous deformation of the porous solid. The creation of new inner solid-fluid interfaces involves changes of the interface energy, whereas the deformation of the porous solid involves only the elastic energy stored by the solid matrix. The Lagrangian saturation is the appropriate state variable describing the interface energy changes, while it is a frozen variable in the expression of the constitutive equations of the porous solid. The distinction between the two mechanisms that the Lagrangian saturation concept permits is promising and has already proved to be successful in the poroelastic analysis of confined crystallization [25, 26].

Based on the Lagrangian saturation concept, the energy approach has provided the natural extension of the standard theory of saturated poroelasticity and poroplasticity to unsaturated conditions. Using mainly macroscopic arguments, the analysis has revealed that the use of the averaged pore pressure as an effective pore pressure may not be general. Its validity was shown to be ultimately based on the assumption of the iso-deformation of the porous networks. The theory has been successfully extended to the case of connected porous networks. As for elastoplastic ordinary solids, the main interest of the approach is to provide a generic framework for recognizing the physical status of each variable and for formulating the constitutive equations of unsaturated elastoplastic porous solids, prior to premature assumptions concerning the poroelastic properties, the plastic yield function, and the flow rule. The specification of the latter, which will involve the microstructure of the porous network, may be worked out by combining adequately the promising approaches offered by micro-poromechanics $[11,36]$ and experimental observations. It is actually hoped that the above developments might usefully drive the experimentalists to pursue a direction where they are most likely to produce new results.

\section{APPENDIX A}

The macroscopic-averaged mean stress $\sigma=\sigma_{k k} / 3$ is the space averaged of the microscopic stress so that we may write

$$
\sigma=\left(1-\phi_{0}\right) \sigma_{\mathrm{s}}-\phi_{0} S_{1} p_{1}-\phi_{0} S_{2} p_{2}
$$

In (A1) the membrane effects associated with the surface energy of the solid-fluid interfaces have been assumed to be negligible. As a result, $p_{J}$ is the pressure actually transmitted to the solid matrix through the internal solid walls delimiting the porous volume that the fluid $J$ occupies. Consider then an experiment where

$$
\sigma=-p, \quad p_{J}=p, \quad \theta=0
$$

A combination of the last two equations provides

$$
\sigma_{\mathrm{s}}=-p
$$

so that the volumetric strain $\varepsilon_{\mathrm{S}}$ related to the solid matrix (assumed to be homogeneous) is

$$
\varepsilon_{\mathrm{S}}=-p / k_{\mathrm{S}}
$$

In the particular experiment defined by the loading condition (A2) we have

$$
\varepsilon=\varphi_{J} /\left(\phi_{0} S_{J}\right)=\varepsilon_{\mathrm{s}}
$$


since the solid matrix is loaded by the same uniform pressure on all its boundaries. Substituting (A5) in (A4), we get

$$
\varepsilon=\varphi_{J} /\left(\phi_{0} S_{J}\right)=-p / k_{\mathrm{s}}
$$

Use of (A6) in (41) or (42), where we let $p_{J}=p$ and $\theta=0$, finally provides the first relation of (44).

The most direct derivation of the second relation of (44) invokes the additive character of entropy. Following [15] the expression of entropy variation $\Sigma-\Sigma_{0}$ of the linear poroelastic solid is

$$
\Sigma-\Sigma_{0}=3 a \sigma+3\left(a b_{1}-a_{1}\right) p_{1}+3\left(a b_{2}-a_{2}\right) p_{2}+\frac{C_{\sigma}}{T_{0}} \theta
$$

where $C_{\sigma}$ is the heat capacity of the porous solid at zero stress and zero pore pressure. The entropy variation of the solid matrix is

$$
\Sigma_{s}-\Sigma_{s}^{0}=3 \alpha_{s} \sigma_{s}+\frac{C_{\sigma_{s}}}{T_{0}} \theta
$$

Substitution of (A1) in (A8) provides

$$
\left(1-\phi_{0}\right)\left(\Sigma_{s}-\Sigma_{s}^{0}\right)=3 \alpha_{s} \sigma+3 \alpha_{s} \phi_{0} S_{1} p_{1}+3 \alpha_{s} \phi_{0} S_{2} p_{2}+\frac{\left(1-\phi_{0}\right) C_{\sigma_{s}}}{T_{0}} \theta
$$

Owing to the additive character of entropy, whatever the values of $\sigma, p_{J}$, and $\theta$ we must have

$$
\Sigma-\Sigma_{0}=\left(1-\phi_{0}\right)\left(\Sigma_{s}-\Sigma_{s}^{0}\right)
$$

A comparison of (A7) and (A9) provides the second relation of (44) and in addition we get the relation $C_{\sigma}=\left(1-\phi_{0}\right) C_{\sigma_{s}}$. Substituting (50) in (44), while using the relations (21) and (27) related to $N$ and $a$, in addition to (50) we finally get

$$
1 / N_{J J}+1 / N_{12}=S_{J} / N, \quad a_{J}=S_{J} a_{\varphi}
$$

Using (A11) and (50) in (41) and (42), irrespective of any temperature variation the iso-deformation assumption (48) finally results in

$$
p_{1}=p_{2}=p: \varphi_{1} / \phi_{0} S_{1}=\varphi_{2} / \phi_{0} S_{2}
$$

\section{APPENDIX B}

When expressing the momentum balance for the mixture, the use of the Eulerian saturation is generally preferred to the use of the Lagrangian saturation. When subsequently addressing the energy and entropy balance this may mislead to the general conclusion that the averaged pressure plays the role of an effective pore pressure [15]. To further explore this point let us here assume that no irreversibility of any kind occurs. The Clausius-Duhem inequality then becomes an equality and energy balance (11) extends in the form

$$
\sigma_{i j} \mathrm{~d} \varepsilon_{i j}+p_{\mathrm{I}} \mathrm{d} \phi_{\mathrm{I}}+p_{\mathrm{II}} \mathrm{d} \phi_{\mathrm{II}}-\mathrm{d} F=0
$$


where we used the indices I and II in order to address simultaneously the case of disconnected networks $(I=1, I I=2)$ and the case of connected porous networks $(I=w F, I I=n w F)$. Updating the notations, we write

$$
\phi_{\mathrm{I}}=\phi s_{\mathrm{I}}, \quad \phi_{\mathrm{II}}=\phi s_{\mathrm{II}}, \quad s_{\mathrm{I}}+s_{\mathrm{II}}=1
$$

and

$$
\phi_{\mathrm{I}}=\phi_{0} S_{\mathrm{I}}+\varphi_{\mathrm{I}}, \quad \phi_{\mathrm{II}}=\phi_{0} S_{\mathrm{II}}+\varphi_{\mathrm{II}}, \quad S_{\mathrm{I}}+S_{\mathrm{II}}=1
$$

Substituting (B2) in (B1) and using (1), we derive

$$
\sigma_{i j} \mathrm{~d} \varepsilon_{i j}+\left(s_{\mathrm{I}} p_{\mathrm{I}}+s_{\mathrm{II}} p_{\mathrm{II}}\right) \mathrm{d} \varphi-\phi\left(p_{\mathrm{II}}-p_{\mathrm{I}}\right) \mathrm{d} s_{\mathrm{I}}-\mathrm{d} F=0
$$

which agrees with the expression derived in $[15,34]$. In the case of an incompressible solid matrix, we have $\mathrm{d} \varepsilon_{k k}=\mathrm{d} \varphi$ and we get

$$
\left[\sigma_{i j}+\left(s_{\mathrm{I}} p_{\mathrm{I}}+s_{\mathrm{II}} p_{\mathrm{II}}\right) \delta_{i j}\right] \mathrm{d} \varepsilon_{i j}-\phi\left(p_{\mathrm{II}}-p_{\mathrm{I}}\right) \mathrm{d} s_{\mathrm{I}}-\mathrm{d} F=0
$$

which agrees with the expression independently derived in [37]. Energy balance (B4) allows us to conclude that

$$
s_{\mathrm{I}} p_{\mathrm{I}}+s_{\mathrm{II}} p_{\mathrm{II}}=\frac{\partial F}{\partial \varphi}, \quad-\phi\left(p_{\mathrm{II}}-p_{\mathrm{I}}\right)=\frac{\partial F}{\partial s_{\mathrm{I}}}
$$

In infinitesimal transformations $\phi$ may be replaced by $\phi_{0}$ in (B5) and we get

$$
s_{\mathrm{I}} p_{\mathrm{I}}+s_{\mathrm{II}} p_{\mathrm{II}}=\frac{\partial F}{\partial \varphi}, \quad-\phi_{0}\left(p_{\mathrm{II}}-p_{\mathrm{I}}\right)=\frac{\partial F}{\partial s_{\mathrm{I}}}
$$

According to the first equation of (B6) or (B7), the pressure $s_{I} p_{\mathrm{I}}+s_{\mathrm{II}} p_{\mathrm{II}}$ is the energy conjugate variable of the overall porosity change $\varphi$ as the pore pressure $p$ does in saturated conditions. It is thereby tempting to conclude on general grounds that $s_{\mathrm{I}} p_{\mathrm{I}}+s_{\mathrm{II}} p_{\mathrm{II}}$ plays the role of an effective pore pressure and that the second equation in (B7) is the state equation governing the capillary invasion of the porous solid, resulting in

$$
F=F_{\mathrm{s}}\left(\varepsilon_{i j}, \varphi, \chi\right)+\phi_{0} U\left(s_{\mathrm{I}}\right)
$$

where $F_{\mathrm{S}}\left(\varepsilon_{i j}, \varphi, \chi\right)$ stands for the free energy (15) of the porous solid related to the saturated case, and where $U\left(s_{\mathrm{I}}\right)$ accounts for the change of the energy of the solid-fluid interface. Unfortunately, this cannot constitute a general approach for the simple reason that the second equation of (B7), when combined with (B8), is unsuitable for the case of disconnected networks where no capillary invasion occurs so that no change in the interface energy is involved.

To make the link between the state equation formulation (B7) with the formulation carried out in the main text, let us restrict to infinitesimal transformations where $\varphi$ and $\varphi_{\text {I or II }}$ are infinitesimal quantities. From (B2) and (B3) we then derive

$$
\varphi_{\mathrm{I}} \simeq \phi_{0}\left(s_{\mathrm{I}}-S_{\mathrm{I}}\right)+\varphi S_{\mathrm{I}}, \quad \varphi_{\mathrm{II}} \simeq \phi_{0}\left(s_{\mathrm{II}}-S_{\mathrm{II}}\right)+\varphi S_{\mathrm{II}}
$$

and we write

$$
F_{\mathrm{S}}=F_{\mathrm{S}}\left(\varepsilon_{i j}, \varphi_{\mathrm{I}}=\phi_{0}\left(s_{\mathrm{I}}-S_{\mathrm{I}}\right)+\varphi S_{\mathrm{I}}, \varphi_{\mathrm{II}}=\phi_{0}\left(s_{\mathrm{II}}-S_{\mathrm{II}}\right)+\varphi S_{\mathrm{II}}, \chi\right)
$$


In the case of disconnected networks $(\mathrm{I}=1, \mathrm{II}=2)$ the Lagrangian saturations $S_{\mathrm{I}}$ and $S_{\mathrm{II}}$ are constant whereas the Eulerian saturations $s_{\mathrm{I}}$ and $s_{\mathrm{II}}$ are not. Letting $F=F_{\mathrm{S}}$ in state equations (B7), the latter are then easily recognized to be an alternative, although abstruse, formulation of state equations (15). In the case of connected networks $(I=w F, I I=n w F)$, formulation (B7) of the state equation does not derive benefit from expression (63) of $F$, so that the formulation is not operational since the key relations (64) are lost. Besides the iso-deformation assumption (48) amounts to writing

$$
\varphi_{\mathrm{I}} / S_{\mathrm{I}}=\varphi_{\mathrm{II}} / S_{\mathrm{II}}=\varphi
$$

This results in $s_{\mathrm{I}}-S_{\mathrm{I}}=s_{\mathrm{II}}-S_{\mathrm{II}}=0$ in (B9). As a result, instead of having to consider both $\varphi_{\mathrm{I}}$ and $\varphi_{\mathrm{II}}$ as independent variables, only the overall porosity change $\varphi$ is involved in the expression (B10) of $F_{\mathrm{s}}$. Equation (B10) then reduces to the form (B8). As a conclusion (B8) turns out to be an alternative way of formulating the iso-deformation assumption (B11) but does not derive from (B7).

\section{ACKNOWLEDGEMENTS}

The author is indebted to Carlos Santamarina for insightful discussions during a one-week invitation at Georgiatech University in the Spring 2006.

\section{REFERENCES}

1. Terzaghi K. Theoretical Soil Mechanics. Wiley: New York, 1943.

2. Biot MA. General theory of three dimensional consolidation. Journal of Applied Physics 1941; 12:155-164.

3. Powers T, Helmuth RA. Theory of volume changes in hardened Portland cement paste during freezing. Proceedings, Highway Research Board, Portland Cement Association 1953; 32(33):286-297.

4. Bowen RM. Theory of mixtures. In Continuum Physics, Eringen AC (ed.), vol. 3. Academic Press: New York, 1976; 1-127.

5. Hassanizadeh M, Gray WG. General conservation equations for multi-phase systems: 1. Averaging procedures. Advances in Water Research 1979; 2:131-144. 2. Mass, momenta, energy, and entropy equations. Advances in Water Research 1979; 2:191-203.

6. Prévost J-H. Mechanics of continuous porous media. International Journal of Engineering Science 1980; 18: 787-800.

7. Bear J, Bachmat Y. Introduction to Modeling of Transport in Porous Media. Kluwer: Amsterdam, 1990.

8. Lewis RW, Schrefler BA. The Finite Element Method in the Static and Dynamic Deformation and Consolidation of Porous Media (2nd edn). Wiley: Chichester, 1998.

9. de Boer R. Theory of Porous Media. Verlag/Hersteller, Springer: Berlin, 2000.

10. Borja RI. On the mechanical energy and effective stress in saturated and unsaturated porous continua. International Journal of Solids and Structures 2006; 43:1764-1786.

11. Dormieux L, Kondo D, Ulm F-J. Microporomechanics. Wiley: Chichester, 2006.

12. Biot MA. Variational Lagrangian-thermodynamics of non isothermal finite strain. Mechanics of porous solid and thermomolecular diffusion. International Journal of Solids and Structures 1977; 13:579-597.

13. Coussy O. Thermodynamics of saturated porous solids in finite deformation. European Journal of Mechanics A/Solids 1989; 8:1-14.

14. Coussy O. Mécanique des Milieux Poreux. Technip: Paris, 1991 (In English, Mechanics of Porous Continua. Wiley: Chichester, 1995).

15. Coussy O. Poromechanics. Wiley: Chichester, 2004.

16. Coussy O, Detournay E, Dormieux L. From mixture theories to Biot's theory. International Journal of Solids and Structures 1998; 35:4619-4635.

17. Detournay E, Cheng AH-D. Fundamentals of poroelasticity. In Comprehensive Rock Engineering, Hudson J (ed.), vol. II. Pergamon Press: Oxford, 1994; 113-169. 
18. Wang HF. Theory of Linear Poroelasticity. Princeton Series in Geophysics. Princeton University Press: Princeton, NJ, 2000.

19. Coussy O. A general theory of thermoporoelastoplasticity for saturated materials. Transport in Porous Media 1989; 4:281-293.

20. Bishop AW, Blight GE. Some aspects of effective stress in saturated and partly saturated soils. Géotechnique 1963; 13:177-197.

21. Hutter KL, Laloui L, Vulliet L. Thermodynamically based mixture models of saturated and unsaturated soils. Mechanics of Cohesive-Frictional Materials 1999; 4(4):295-338.

22. Gray WG, Schrefler BA. Thermodynamic approach to effective stress in partially saturated porous media. European Journal of Mechanics - A/Solids 2001; 20(4):521-538.

23. Loret B, Khalili N. An effective stress elastic-plastic model for unsaturated porous media. Mechanics of Materials 2002; 34:97-116.

24. Wheeler SJ, Sharma RJ, Buisson MSR. Coupling of hydraulic hysteresis ans stress-strain behaviour in unsaturated soils. Géotechnique 2003; 53(1):41-54.

25. Coussy O. Poromechanics of freezing materials. Journal of the Mechanics and Physics of Solids 2005; 53: $1689-1718$.

26. Coussy O. Deformation and stress from drying-induced crystallization of salt. Journal of the Mechanics and Physics of Solids 2006; 54:1517-1547.

27. Alonso EE, Gens A, Josa A. A constitutive model for partially saturated soils. Géotechnique 1990; 40(3):405-430.

28. Gibbs JW. On the Equilibrium of Heterogeneous Substances. Equilibre des Systèmes Chimiques, Carré G, Naud C (eds), Paris, 1899, Editions Pierre Gabay: Paris, reprint 2003 (translated in French by H. Le Châtelier).

29. Duhem P. Traité d'Energétique ou de Thermodynamique Générale, Tomes I et II. Gauthier-Villars: Paris, 1911. Editions Pierre Gabay: Paris, reprint 1997.

30. Collins IF. The concept of stored plastic work or frozen elastic energy in soil mechanics. Géotechnique 2005; 55(5):373-382.

31. Chateau X, Dormieux L. Micromechanics of saturated and unsaturated porous media. International Journal of Numerical and Analytical Methods in Geomechanics 2002; 26:831-844.

32. Gallipoli D, Gens A, Sharma R, Vaunat J. An elasto-plastic model for unsaturated soil incorporating the effects of suction and degree of saturation on mechanical behaviour. Géotechnique 2003; 53(1):123-135.

33. Coussy O, Monteiro P. Unsaturated poroelasticity for crystallization in pores. Computers and Geotechnics 2007, in press. doi:10.1016/j.compgeo.2007.02.007

34. Dangla P, Malinsky L, Coussy O. Plasticity, imbibition-drainage curves for unsaturated soils: an unified approach. NUMLOG VI, 6th International Symposium on Numerical Methods in Geomechanics, Montréal, 2-4 July 1997; 141-146.

35. Buisson MSR, Wheeler SJ. Inclusion of hydraulic hysteresis in a new elasto-plastic framework for unsaturated soils. In Experimental Evidence and Theoretical Approaches for Unsaturated Soils, Tarantino A, Mancuso C (eds). Rotterdam: Balkema, 2000; 109-119.

36. de Buhan P, Dormieux L. On the validity of the effective stress concept for assessing the strength of saturated porous materials: a homogenization approach. Journal of the Mechanics and Physics of Solids 1996; 44(10): $1649-1667$.

37. Houlsby GT. The work input to an unsaturated granular material. Géotechnique 1997; 47(1):193-196.

Copyright (C) 2007 John Wiley \& Sons, Ltd.

Int. J. Numer. Anal. Meth. Geomech. 2007; 31:1675-1694

DOI: $10.1002 / \mathrm{nag}$ 